12

\title{
Расчет трекинг-силы при развитии резистивной шланговой неустойчивости релятивистского электронного пучка
}

\author{
(C) Е.К. Колесников, А.С. Мануйлов, В.С. Петров \\ Санкт-Петербургский государственный университет, \\ 199034 Санкт-Петербург, Россия \\ ฯ e-mail: a.manuylov@spbu.ru, man06@mail.ru
}

(Поступило в Редакцию 25 января 2017 г.)

Исследована трекинг-сила, действующая на релятивистский электронный пучок со стороны омического плазменного канала достаточно малой проводимости. Показано, что при заданном характере развития резистивной шланговой неустойчивости вдоль импульса пучка данная сила существенно зависит от частоты и инкремента нарастания рассматриваемой неустойчивости.

DOI: 10.21883/JTF.2018.01.45492.2182

\section{Введение}

Определенный интерес в рамках задачи распространения релятивистских электронных пучков (РЭП) в плотных газоплазменных средах представляет исследование устойчивой проводки пучков по предварительно созданным омическим плазменным каналам [1-11]. В работе [5] была предложена модель для расчета трекинг-силы, действующей на РЭП, испытывающий поперечные смещения и распространяющийся внутри однородного по радиусу омического плазменного канала низкой проводимости. В этом случае основную роль играют электростатические силы взаимодействия отклоненного в поперечном направлении пучка и генерированным на границе плазменного канала дипольным возмущенным зарядом. Однако в [5] не был проведен численный анализ зависимости трекинг-силы от скорости нарастания амплитуды шлангового отклонения пучка и от временной частоты изменения указанной амплитуды. Этим вопросам и посвящена настоящая работа.

\section{Постановка задачи}

Рассмотрим параксиальный моноскоростной в продольном направлении азимутально-симметричный РЭП, распространяющийся вдоль оси $z$ цилиндрической системы координат $(r, \theta, z)$ вдоль предварительно созданного омического плазменного канала низкой скалярной проводимости $\sigma_{c h}$, когда выполнено условие $4 \pi \sigma_{c h} R_{b 0} / c \leq 1$, где $R_{b 0}$ - характерный радиус пучка, $c$ - скорость света. Будем предполагать, что проводимость $\sigma$ постоянна до некоторого радиуса $R_{c h}$ и равна $\sigma_{c h}$, а при $r>R_{c h}$ равна нулю. Предполагается, что при шланговых поперечных отклонениях пучок не выходит за пределы плазменного канала. При этом полагается, что динамика резистивной шланговой неустойчивости (РШН) ограничена только линейным режимом, когда выполнено соотношение $Y / R_{b 0} \ll 1$, здесь $Y-$ амплитуда поперечных отклонений оси симметрии пучка при развитии указанной неустойчивости.

В работе [5] было получено уравнение динамики РШН РЭП в омическом плазменном канале низкой проводимоси, которое является электростатическим аналогом, соответствующим уравнению РШН в режиме высокой проводимости предварительно созданного омического плазменного канала [2,3]. Указанное уравнение имеет вид

$$
\frac{\partial^{2} Y}{\partial z^{2}}=\frac{\pi}{I_{b} I_{\mathrm{A}}} \int_{0}^{\infty} d r r\left(Y \frac{\partial \rho_{b 0}}{\partial r} \frac{\partial \Phi_{0}}{\partial r}-\frac{\rho_{b 0}}{r} \frac{\partial}{\partial r}\left(r \Phi_{1}\right)\right)
$$

где $I_{b}$ - полный ток пучка, $I_{\mathrm{A}}$ - предельный ток Альфвена, $\rho_{b 0}-$ невозмущенная плотность заряда пучка, $\Phi_{0}$ и $\Phi_{1}-$ соответственно невозмущенный и возмущенный скалярный электрический потенциал электромагнитного коллективного поля системы плазма-пучок.

С помощью выделения вклада дипольного заряда на границе однородного плазменного канала в уравнение (1), решения возмущенного решения Пуассона для потенциала $\Phi_{1}$ и условия временного изменения дипольного заряда при $r=R_{c h}$ за счет омического тока в возмущенном электростатическом поле по аналогии с результатами работы [5] мы формулируем выражение для трекинг-силы, обусловленной действием дипольного заряда на границе плазменного канала, действующего на смещенный в поперечном направлении РЭП в следующем виде:

$$
F_{t r}=-\frac{2 I_{b 0}}{I_{\mathrm{A}}} \frac{R_{b 0}}{R_{c h}^{2}} G(\xi)
$$

где $I_{b 0}-$ амплитуда полного тока пучка, $I_{\mathrm{A}}-$ предельный ток Альфвена, $R_{c h}-$ характерный радиус плазменного канала, $R_{b 0}-$ характерный радиус пучка, $\xi=v_{z} \tau, \tau=t-z / v_{z}-$ сдвинутое время, $t-$ время, $z$ - дистанция, пройденная пучком, $v_{z}-$ продольная компонента скорости электронов пучка. Функция $G(\xi)$ 

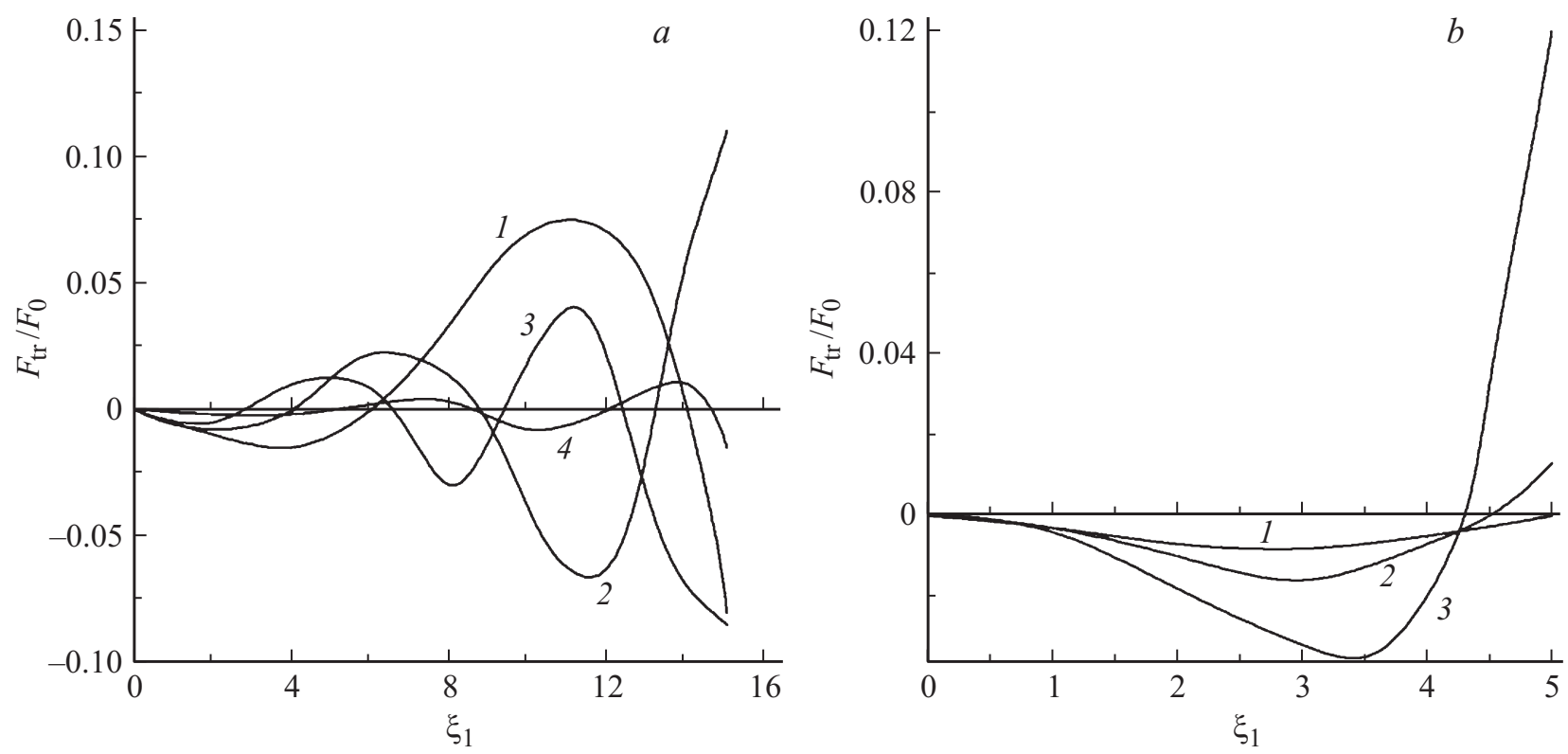

Зависимость безразмерной силы $F_{t r} / F_{0}=-G F_{t r} / F_{0}=-G\left(F_{0}=2 I_{b 0} R_{b 0} /\left(I_{\mathrm{A}} R_{c h}^{2}\right)\right.$ - характерная трекинг-сила) от координаты $\xi_{1}$ : $a$ - кривая 1 соответствует частоте $\omega=0.4,2-0.7,3-1,4-4 ; b-$ кривая 1 соответствует параметру $a=0.2,2-0.6$, $3-1$.

имеет вид

$$
\begin{gathered}
G\left(\xi_{1}, z_{1}\right)=\int_{-\infty}^{\xi_{1}} d \xi_{1}^{*}\left[\exp \left(\frac{\xi_{1}^{*}-\xi_{1}}{2}\right)-\exp \left(\xi_{1}^{*}-\xi_{1}\right)\right] \\
\times\left\{\frac{\partial f\left(\xi_{1}^{*}\right)}{\partial \xi_{1}^{*}} Y_{1}\left(z_{1}, \xi_{1}^{*}\right)+\frac{\partial Y_{1}\left(z_{1}, \xi_{1}^{*}\right)}{\partial \xi_{1}^{*}} f\left(\xi_{1}^{*}\right)\right\},
\end{gathered}
$$

$\xi_{1}=\xi / \xi_{c}, \quad \xi_{c}=1 /\left(4 \pi \sigma_{c h}\right)-$ характерный пространственный масштаб зарядовой нейтрализации, $f\left(\xi_{1}\right)-$ функция модуляции тока, $Y_{1}\left(z_{1}, \xi_{1}\right)=Y\left(z_{1}, \xi_{1}\right) / R_{b 0}$, где $Y\left(z_{1}, \xi_{1}\right)$ - амплитуда поперечного смещения оси симметрии тонкого поперечного сегмента пучка, расположенного на безразмерном расстоянии $\xi_{1}$ от фронта РЭП и прошедшего дистанцию $z \quad\left(z_{1}=z k_{S}\right.$, где $k_{S}=\left(I_{b} / I_{\mathrm{A}}\right)^{1 / 2} / R_{b 0}$ - бетатронное волновое число).

После ряда преобразований получим

$$
\begin{aligned}
G\left(\xi_{1}, z_{1}\right)= & -\int_{-\infty}^{\xi_{1}} d \xi_{1}^{*}\left[0.5 \exp \left(\frac{\xi_{1}^{*}-\xi_{1}}{2}\right)\right. \\
& \left.-\exp \left(\xi_{1}^{*}-\xi_{1}\right)\right] f\left(\xi_{1}^{*}\right) Y_{1}\left(z_{1}, \xi_{1}^{*}\right) .
\end{aligned}
$$

Предположим, что РШН пучка развивается вдоль его импульса по закону

$$
Y_{1}\left(z_{1}^{*}, \xi_{1}\right)=Y_{0} \exp \left(a \xi_{1}\right) \cos \left(\omega \xi_{1}\right),
$$

где $Y_{0}-$ начальная амплитуда неустойчивости, $a-$ инкремент нарастания РШН вдоль импульса пучка, $\omega-$ временная частота колебаний при развитии неустойчивости, $z_{1}^{*}$ - некоторая фиксированная дистанция от ускорителя.
На рисунке $a$ представлена зависимость безразмерной силы $F_{t r} / F_{0}=-G\left(F_{0}=2 I_{b 0} R_{b 0} /\left(I_{\mathrm{A}} R_{c h}^{2}\right)-\right.$ характерная трекинг-сила) от координаты $\xi_{1}$ при разных значениях временно́й частоты $\omega$ в случае, когда $a=0.2, Y_{0}=0.02$ и $f\left(\xi_{1}\right)=1$. Как следует из рисунка, временна́я частота развития РШН существенно влияет как на величину силы, так и на дапазоны координаты $\xi_{1}$, где сила меняет знак и, например, из трекинг-силы (отрицательное значение $\left.F_{t r} / F_{0}\right)$ становится детрекинг-силой (положительное значение $\left.F_{t r} / F_{0}\right)$.

На рисунке $b$ представлена зависимость безразмерной силы $F_{t r} / F_{0}$ от координаты $\xi_{1}$ при разных значениях параметра $a$ (инкремента нарастания РШН по координате $\left.\xi_{1}\right)$ и при частоте $\omega=0.5$. Остальные параметры совпадают со случаем рисунка $b$. Из рисунка следует, что при малых $\xi_{1}$ сила отрицательна и является трекингсилой, а затем меняет знак. Кроме того, с увеличением параметра $a$ наблюдается нарастание силы пучковоканального взаимодействия.

\section{Заключение}

С помощью численного анализа исследована сила, действующая на РЭП, испытывающий поперечные колебания при развитии РШН пучка при его распространении в омическом плазменном канале достаточно малой проводимости. При заданной амплитуде поперечных колебаний пучка изучено влияние временно́й частоты неустойчивости и инкремента нарастания вдоль импульса пучка. Как показал численный расчет, временна́я частота развития шланговой неустойчивости существенно влияет как на величину указанной силы, так и 
на диапазоны координаты (характеризующей расстояние поперечного сечения пучка от его переднего фронта), в пределах которых исследуемая сила меняет знак. Кроме того, исследовано влияние величины инкремента нарастания РШН на знак и величину изучаемой силы. С увеличением указанного инкремента величина силы нарастает и в головной части РЭП становится возвращающей пучок к оси плазменного канала (трекинг-эффект), а при более значительных расстояниях от фронта пучка данная сила работает на выталкивание РЭП из канала (детрекинг-эффект). Это приводит к тому, что головная часть пучка будет удерживаться в канале, а более дальние от фронта сегменты пучка будут выбрасываться из канала. Такая картина наблюдается при всех инкрементах нарастания РШН, рассмотренных при численном анализе задачи.

\section{Список литературы}

[1] Рухадзе А.А., Богданкевич Л.С., Росинский С.Е., Рухлин В.Г. Физика сильноточных релятивистских электронных пучков. М.: Атомиздат, 1980. 167 с.

[2] Миллер $P$. Введение в физику сильноточных пучков заряженных частиц. М.: Мир, 1984. 432 с.

[3] Колесников Е.К., Мануйлов А.С., Филиппов Б.В. Динамика пучков заряженных частиц в газоплазменных средах. СПб.: Изд-во СПбГУ, 2002. 98 с.

[4] Колесников Е.К., Мануйлов А.С., Зеленский А.Г. Динамика релятивистских электронных пучков в режиме ионной фокусировки. Воскресенск: Изд-во Позитив, 2013. 104 с.

[5] Lee E.P. // Livermore Lab. Report UCID - 19674. 1983. P. 10.

[6] Welch D.R., Bieniosek F.M., Godfrey B.B. // Phys. Rev. Lett. 1990. Vol. 65. N 25. P. 3128-3131.

[7] Hui B., Lampe M. // V Intern. Conf. High Power Particle Beams. San Francisco. 1983. P. 374-377.

[8] Мануйлов А.С. // ЖТФ. 2013. Т. 83. Вып. 10. С. 151-154.

[9] Колесников Е.К., Мануйлов А.С. // ЖТФ. 2000. Т. 70. Вып. 5. С. 68-73.

[10] Колесников Е.К., Мануйлов А.С. // ЖТФ. 1997. Т. 67. Вып. 6. С. 69-71.

[11] Mamedov B.A. // Astropart. Phys. 2015. Vol. 64. P. 1-3. 